\title{
Fundamentos de las normas de descripción archivística y bibliotecaria
}

\author{
Carlos Oliva Marañón \\ Universidad Rey Juan Carlos - URJC, España.
}

\section{ANÁLISIS}

\begin{abstract}
Resumen
Los archivos, las bibliotecas y los centros de documentación han aumentado su visibilidad en Internet durante los últimos años para interactuar con los usuarios y aproximarles a los fondos documentales en formato digital. La normalización es toda actividad colectiva dirigida a establecer e implementar normas para definir los requisitos que deben cumplir bienes, servicios y procedimientos. Esta investigación desarrolla las principales normas de descripción archivística (NEDA, ISAD) y bibliotecaria (ISBD, MARC). Este proceso se considera una operación de análisis documental que respeta el principio de procedencia. Las especiales características del documento de archivo; la adaptación de las normas al fondo documental pertinente; la dificultad de la coherencia y la cohesión normativa; el aumento de volumen de trabajo; el alto coste económico; y la gestión del tiempo constituyen los principales inconvenientes para satisfacer adecuadamente las necesidades de información de los usuarios.
\end{abstract}

Palabras clave

Descripción archivística ; Descripción bibliotecaria ; Normalización ; Normas de descripción ; Análisis documental ; Necesidades de información ; Documentación digital

\section{Basis for archival and librarian description standards}

\begin{abstract}
The archives, libraries and documentation centers have increased their visibility on the Internet in recent years to interact with users and them closer to the documentary in digital format. Standardization is any collective activity aimed at establishing and implementing standards to define requirements for goods, services and procedures. This research develops the main archival description standards (NEDA, ISAD) and librarian (ISBD, MARC). This process is considered a documentary analysis operation that respects the principle of provenance. The special characteristics of the archive document, the adaptation of the rules relevant to the document collection, the difficulty of coherence and cohesion policy, the increased volume of work, the high cost and time management are the main drawbacks to adequately meet the information needs of users.
\end{abstract}

Keywords

Archival description ; Librarian description ; Normalization ; Description standards ; Document analysis ; Information needs ; Digital documentation

\section{Introducción}

El modo en que se describen los documentos depende en gran medida de la forma en que se conciben los objetivos del servicio de archivo. Los sistemas de archivo se pueden concebir como sistemas complejos que pueden abarcar todas la etapas del ciclo de vida de los documentos y que permiten cumplir objetivos de carácter administrativo y cultural al mismo tiempo. Igualmente, el modo de descripción de los documentos depende también de las herramientas tecnológicas disponibles. En estos momentos se asiste a una transformación del modelo informativo de carácter jerárquico a otro basado en métodos relacionales derivado, en gran medida, de la importación por parte de la archivística de ciertas técnicas de diseño y análisis de bases de datos. 
Sin duda, esa adaptación implica una revisión de las posiciones teóricas de esta disciplina en la medida en que estas son siempre una respuesta a necesidades prácticas (Barbadillo Alonso, 2011).

La historia del proceso de normalización internacional de la descripción de documentos de archivo se inicia en 1998, en un encuentro de expertos convocado en Otawa por los Archivos Nacionales de Canadá y el Consejo Internacional de Archivos sobre esta materia. Los asistentes reconocieron por unanimidad la necesidad de una norma internacional de descripción archivística y solicitaron que se formara un grupo de trabajo con esta finalidad. Los temas que estuvieron presentes en este comienzo del proceso de normalización han mantenido su protagonismo en años posteriores: las normas como instrumento para garantizar el intercambio de información entre centros de archivo, para permitir la creación de sistemas unificados de información archivística y para facilitar el tratamiento informático de las descripciones.

El proceso de normalización de la descripción archivística, iniciado en Estados Unidos a principios de los años 80, ha influido notablemente en el cambio del propio concepto de descripción. Mientras que tradicionalmente se equiparaba la descripción al proceso de elaboración de instrumentos de descripción (guías, inventarios y catálogos), hoy la descripción se considera una operación en sí misma, una operación de análisis documental encaminada a la creación de representaciones exactas y concisas de cualquier unidad archivística, siempre bajo el respeto del principio de procedencia.

El interés por la normalización no es, sin embargo, algo reciente. Ya Felipe II, en su Instrucción para el Gobierno del Archivo de Simancas de 1588, al hablar de la realización del libro de los inventarios, afirmaba que "las personas que adelante succedieren en el dicho cargo de archivero an de tener particular cuydado de hacer lo mismo cada uno en su tiempo". En época más reciente, en la inauguración del primer Congreso Nacional de Archivos, celebrado en Sevilla en 1963, Antonio Matilla Tascón destacaba la conveniencia de normalizar en los archivos al aseverar que "los archiveros no pueden ser la excepción en la actividad de la vida presente, que exige, en todas las funciones, aparte de una mutua coordinación, la racionalización de los procesos y la normalización de las formas. Con ello se simplifican los métodos, se ahorra trabajo y tiempo en la ejecución y se obtienen mayores garantías en los resultados" (Bonal Zazo, 2000).

A principios de la década de los noventa, el prestigioso archivero francés Michel Duchein afirmaba: "se plantea a veces la cuestión: ¿sería posible hacer normas de carácter internacional para la inventariación de archivos? Mi contestación sería: no, en ningún caso. Normas de edición, sí. Quizás, normas de indización. Pero, bajo ningún concepto, normas análogas a la existentes para la catalogación de libros". En el XII Congreso Internacional de Archivos, celebrado en Montreal en 1992, María Guercio planteaba la siguiente pregunta: "what are the concrete reasons why archivists have had difficulties in the past in creating and accepting standards? (Guercio, 1994, p. 201)". Las dificultades han sido numerosas, todas ellas caracterizadas por unas particularidades comunes, derivadas del carácter singular de los archivos y de la teoría archivística, frente a otros centros documentales.

La primera dificultad destacable es el propio objeto de los archivos y de la Archivística: el documento. Señala Pedro González que "el carácter único del documento de archivo, frente al libro o la revista, ha determinado parcialmente el rechazo a las normas de descripción y ha llevado, en consecuencia, a la creación y utilización de normas específicas en cada archivo" (González, 1991). No es, sin embargo, la unicidad documental el único factor que ha determinado el alejamiento de las normas.

\section{Estado de la cuestión}

Los documentos de archivo no son documentos independientes sino que, al contrario, están fuertemente relacionados con sus productores, con las funciones por las que fueron creados y con su propia organización interna. Esta circunstancia, relacionada directamente con la base de la teoría archivística, influye notablemente en todas las operaciones relacionadas con su tratamiento. Por otra parte, las características descritas se ven complicadas por dos factores: en primer lugar, por el creciente volumen documental y la diversificación de sus formatos de presentación (Cox, 1994, p.170) y, en segundo lugar, por la heterogeneidad de los documentos de archivo, como consecuencia del elevado número de productores y de su diversidad a lo largo del tiempo (Guercio, 1994, p. 201). 
Junto a los documentos, también los usuarios han influido negativamente en el desarrollo de prácticas normalizadas. El prototipo convencional de usuario de archivo ha sido, durante mucho tiempo, el del investigador caracterizado por su erudición y amplio conocimiento de la materia estudiada, y también por su laboriosidad y paciencia en el trabajo, pero, sobre todo, como señala Xavier Tarraubella, porque: "No es refia de la informació o l'exhaustivitat dels inventaris realizats pels arxivers i vol accedir directament a la documentació arxivada, a las sèries documentals, sense la intermediació dels instruments de descripció" (Tarraubella, 1998). Se trataba, por tanto, de usuarios que apenas necesitaban de los archivos poco más que orden y conservación.

A partir de los años 50 comienza a producirse un cambio trascendental en la tipología de usuarios debido, fundamentalmente, al aumento del número de personas que acuden a consultar documentos, lo que provocó una diversificación de los perfiles de usuarios. Esta diversidad repercutirá directamente en la obligación de unificar prácticas y mejorar procedimientos de descripción, capaces de satisfacer todas las necesidades informativas de los usuarios (Bonal Zazo, 2000).

Otro de los factores que ha incidido negativamente en la difusión y utilización de prácticas normativas ha sido la tradicional forma de trabajo del archivero, generalmente aislada, independiente y con unos criterios propios adaptados a sus necesidades específicas (Cox, 1994, p. 173). Causas diversas como el aumento del número de profesionales, la diversificación de su perfil, la proyección social de los archivos y, sobre todo, la influencia de disciplinas afines han contribuido a la introducción de prácticas cooperativas en el ámbito de los archivos, prácticas que exigen el desarrollo de actividades normalizadas.

Otra causa que ha repercutido en la falta de normalización ha sido la diversidad existente en el ámbito de la Archivística, diversidad que se advierte tanto en cuanto a estructuras de organización y funcionamiento de archivos (Muñoz Feliu, 1995, p. 26), como en la existencia de diferentes realidades históricas, administrativas, culturales y de formación (Guercio, 1994, p. 201). Junto a los factores enunciados, todos ellos de carácter teórico o profesional, cabe destacar también la existencia de razones económicas.

La creación de normas técnicas exige contar con un fuerte respaldo económico. Frente a este requisito, la profesión archivística se ha caracterizado, tradicionalmente, por la falta de recursos (Cox, 1994, p. 173). En el ámbito concreto de la descripción archivística existen varias propuestas de clasificación. Apunta González (1992, p. 162), siguiendo en buena medida a los autores norteamericanos, que la normalización de la descripción de archivos puede ser analizada desde diferentes puntos de vista:

- Documentos que permiten normalizar la estructura de los datos (responden a la pregunta: ¿cuáles son los elementos de la descripción?)

- Documentos para la normalización del contenido de cada uno de los elementos de la descripción (dan respuesta a las siguientes preguntas: ¿qué información incluir en cada elemento? y ¿de qué forma?)

- Documentos para la normalización de los instrumentos de descripción.

- Documentos normativos en el área de los lenguajes de indización.

El Grupo de Trabajo sobre Normas de Descripción de la Society of American Archivists (SAA) estableció la existencia de varios tipos de normas, en función de tres criterios diferentes (Bearman, 1989): el poder normativo del texto, su origen y la profundidad en la normalización de la descripción.

Por lo que respecta al primer aspecto, el poder preceptivo de las normas de descripción, es posible establecer la existencia de tres tipos de documentos normativos (Bonal Zazo, 2000): 
- Normas técnicas. Se trata de normas que comúnmente regulan el uso y la compatibilidad de equipamientos y que, en el caso de las Ciencias de la Información, se usan para regular el intercambio de información (Cox, 1994, p. 166). Son las normas más rígidas que, según Crawford,: "is not subject to unilateral change without notice and which, if properly followed, will yield consistent results" (Crawford, 1986). Aunque estas normas regulan numerosos elementos del trabajo cotidiano, incluido el trabajo archivístico (códigos de caracteres, sistemas informáticos, etc.), todavía no se ha desarrollado una norma de este tipo específica para el trabajo archivístico, aunque sí existen adaptaciones de normas técnicas generales procedentes de otros ámbitos (Working Group..., 1989, pp. 456-461).

- Normas profesionales (convenciones o reglas). Las normas profesionales son normas que regulan prácticas y procedimientos de trabajo. Se trata de normas más flexibles que las anteriores y que se acomodan mejor a las prácticas locales. No obstante, pueden tener el mismo valor normativo que las normas técnicas si se aplican estrictamente (Ibídem, p. 167). En este caso, a diferencia de las anteriores, sí que existen normas creadas específicamente para la descripción archivística, tales como la ISAD(G), RDDA, MAD2, APPM e ISAAR (CPF). Tradicionalmente han sido consideradas por los archiveros como las normas más importantes y también las más deseadas.

- Directrices. Se trata de instrucciones generales o recomendaciones que no tienen el mismo valor normativo que las anteriores. Nancy Bolt establece que la diferencia de las directrices con los documentos normativos anteriores es: "there are consequences of not meeting" (Bolt, 1988). Tal como afirma Cox, "las directrices son un paso previo para el desarrollo de normas más específicas (profesionales o técnicas). No obstante, frecuentemente, es difícil establecer la frontera entre unas y otras".

En cuanto a su origen, se puede hablar de dos tipos de normas: normas externas, es decir, normas procedentes de ámbitos de trabajo diferentes del propiamente archivístico; y normas internas, normas creadas específicamente para el desarrollo de trabajos de archivos (Working Group..., 1989, pp. 456-461). Normas como el formato USMARC AMC, RDDA, MAD2, ISAD(G) o APPM son normas de carácter interno, mientras que otras, como las AACR2, son normas externas. No obstante, no se puede establecer una división tajante entre normas externas y normas internas: algunas, como APPM, aunque son de carácter interno, tienen una fuerte influencia de disciplinas externas a la Archivística (Bonal Zazo, 2000)

Dependiendo de la profundidad alcanzada en la normalización existen, según David Bearman, cuatro tipos de normas (Bearman, 1989):

- Normas sobre sistemas de información. Son las más generales, ya que definen las funciones de cada componente dentro de un sistema de información global, tanto si es un solo archivo como si es una red o un sistema de archivos.

- Normas de estructura de datos. Se trata de normas que definen los elementos informativos que deben ser recogidos en los componentes de un sistema de información. Este tipo de normas sólo regulará los datos que es necesario incluir en un sistema de información, pero no su contenido; son normas relativas al formato externo de los datos. Un claro ejemplo de este tipo de normas es el formato USMARC AMC.

- Normas de contenido de datos. Son las normas que regulan qué información introducir en cada elemento definido en las normas de estructura de datos. Son normas tales como las AACR2, APPM, MAD2, ISAD(G) o ISAAR(CPF) (Weber, 1989, pp. 509-510).

- Normas sobre el valor de los datos. Proporcionan listas o tablas de términos, nombres, códigos alfanuméricos $u$ otros datos usados en elementos particulares de estructuras de datos. Se trata de todas aquellas listas de materias, tesauros, listas de autoridades, listas 
de nombres propios, etc., que recogen la forma, ya establecida, de los términos que pueden ser usados para la descripción, sin dar lugar a la interpretación.

Como señala María Guercio, las dificultades para normalizar los datos con la finalidad de crear sistemas de información archivística se encuentran no sólo en el nivel de contenido de datos, sino también en el de estructura de datos (Guercio, 1994). Un programa general de descripción debe abarcar, por tanto, las distintas categorías apuntadas. La justificación de la utilidad de las normas se encuentra en su propia naturaleza. Tal como señala García Gutiérrez, una norma siempre debe: "repercutir positivamente en el bienestar y progreso de la sociedad" (García Gutiérrez, 1989, p. 58), las normas son, por esencia, beneficiosas.

Por lo que respecta a la normalización de la descripción, las normas presentan importantes ventajas. En primer lugar, ventajas para los usuarios, debido a que favorecen la comprensión de las descripciones. Ventajas, en segundo lugar, para la teoría archivística, ya que contribuyen a la comunicación y la cooperación entre los archivos, tanto a escala nacional como internacional. En tercer lugar, la aplicación estricta de normas es ventajosa para el acceso a los documentos; y, como consecuencia de todo lo anterior, las normas conllevan ventajas económicas derivadas de la mejora de la calidad de los servicios (Bonal Zazo, 2000).

La principal utilidad de las normas, desde el punto de vista de los usuarios, es la de favorecer la comprensión de las descripciones. Si partimos de la idea de que la descripción es una representación de una unidad documental, convendremos en que esta representación, debe proporcionar información en cantidad suficiente y forma adecuada para que los usuarios decidan si la documentación es relevante para sus necesidades (Szary, 1989, p. 520), siempre respetando tres objetivos básicos: 1) preservar la integridad de los archivos; 2) comunicar la información que contienen; y 3) facilitar el acceso a todos aquéllos que tengan derecho a él (Haworth, 1992, p. 188).

Además de los usuarios, son los propios archivos y archiveros los beneficiarios indirectos de la normalización. La aplicación de las nuevas tecnologías al almacenamiento e intercambio de información facilita la comunicación y la cooperación archivística, pero exige, como contrapartida, la utilización de normas uniformes. Como señala Szary: "the profesion has embarked on a shared enterprise that requires unambiguos communication"(Szary, 1989, p. 520). Las repercusiones de la normalización son notables para el trabajo archivístico, ya que favorece el desarrollo de iniciativas de cooperación no sólo con otros centros de archivos, sino también con otros profesionales de la información, con sus técnicas y sus equipamientos. La utilización de las grandes redes bibliográficas norteamericanas para la difusión de descripciones archivísticas constituye un buen ejemplo de esta utilidad. Se puede afirmar que la normalización contribuye, de este modo, a la universalización de los problemas y de las prácticas archivísticas (Cox, 1994, p. 169), favoreciendo, también, la búsqueda de soluciones globales.

\section{PARES (Portal de Archivos Españoles)}

El paso previo al empleo de la herramienta de gestión de archivos es la selección de los materiales documentales y archivísticos con los que se pretende trabajar. Se propone la preparación del supuesto práctico a partir de la selección de un Fondo, Sección, Serie y Expediente de un Archivo Español. La documentación archivística más accesible es la que puede encontrarse de acceso libre desde el Portal de Archivos Españoles (PARES). Es el portal desarrollado por la Subdirección General de los Archivos Estatales, considerado como el principal proyecto de difusión de la archivística española en la Web. Es importante reseñar que la documentación disponible consta, fundamentalmente, de un valor histórico que da fe de los hechos acontecidos en la historia del país.

PARES consta de diversos métodos de recuperación de la documentación, siempre basándose en el procedimiento archivístico de la identificación de los sujetos productores, el cuadro de clasificación y la descripción archivística a partir de las normas ISAD-G atendiendo en esencia al área de Alcance y Contenido. Las opciones de recuperación de información son las siguientes:

- Búsqueda Sencilla. Permite una recuperación de fondos, series o expedientes a partir de un término o cadena de texto introducida. Distingue la recuperación en la totalidad de los fondos y en documentación digitalizada. 
- Búsqueda Avanzada. Además de las opciones de recuperación de la búsqueda sencilla, añade la consulta por fechas extremas, sujeto productor, el nombre del archivo, signatura e índices.

- Inventario Dinámico. Visualiza los últimos fondos, series o expedientes digitalizados y, por ende, descritos, sin efectuar consulta previa.

\section{Principales normas de descripción archivística}

Los registros de autoridad de archivos se crearon en un principio para documentar el contexto de producción de los documentos de archivo. Para que esta información resulte de utilidad es necesario vincular los registros de autoridad con las descripciones de esos documentos de archivo.

Los registros de autoridad de archivos también se pueden vincular con cualquier otro recurso informativo de interés. Cuando se establecen tales relaciones es importante describir la naturaleza de la relación entre la institución, persona o familia y el recurso relacionado.

\subsection{Las Normas NEDA}

Las NEDA se desarrollan en el contexto del actual Sistema Archivístico Español, surgido a partir de la configuración del Estado de las Autonomías e integrado por diferentes Sistemas (Estatal y Autonómicos) y Redes Archivísticas. Consecuentemente, en el desarrollo de la NEDA debe participar toda la Comunidad Archivística, y debe ser una herramienta normativa consensuada por todas las partes afectadas para que pueda ser asumida por todos los archivos.

a) Objetivos específicos de las NEDA

- Las NEDA deberán proporcionar normas, de aplicación obligatoria, que establezcan los tipos de representaciones o descripciones de las entidades que forman parte de los sistemas de información archivística, así como sus interrelaciones fundamentales, basadas en un modelo conceptual de datos de alto nivel consensuado, que identifique las entidades archivísticas, sus atributos y relaciones.

- Las NEDA deberán facilitar normas, de aplicación obligatoria, relativas a la introducción de datos de descripción en los sistemas de información archivística, es decir: a) Normas de estructura de datos para los distintos tipos de descripciones de las entidades archivísticas, basadas en el mencionado modelo conceptual de datos, suficientemente detalladas pero independientes de cualquier estructura o sintaxis particular de almacenamiento de datos; y b) Normas de contenido de datos para los componentes estructurales fijados en las normas anteriores.

- Las NEDA deberán facilitar reglas, de aplicación opcional o recomendable, relativas a la salida de datos de descripción de los sistemas de información archivística, es decir,: $\quad$ a) Reglas de aplicación opcional de presentación de datos en pantalla o en salida impresa. Por ejemplo, para las descripciones de los documentos de archivo: etiquetado de acuerdo con la estructura en áreas y elementos de la $\operatorname{ISAD}(\mathrm{G}) 2$; orden y puntuación de las ISBD. Por ejemplo, para las descripciones de las instituciones, personas y familias: etiquetado de acuerdo con la estructura en áreas y elementos de la ISAAR(CPF)2; orden y puntuación de las GARR; y b) Recomendaciones de codificación de datos para 
el intercambio o comunicación entre sistemas, empleando diferentes sistemas de metadatos (EAD, EAC, MARC21, Dublin Core).

\section{b) Fases de desarrollo de las NEDA}

Se propone que las NEDA tengan un desarrollo de en 3 fases:

- Fase 1: regulación de las descripciones de documentos de archivo y de las representaciones de instituciones, personas y familias. En esta fase también debería regularse la selección y formalización de determinados nombres geográficos, en tanto en cuanto pueden formar parte de los puntos de acceso de instituciones (bien como componente inicial de nombre de área jurisdiccional, bien como calificador geográfico).

- Fase 2: regulación de las representaciones de funciones y actividades, y de las descripciones de custodios, cuando estén maduros los estándares internacionales sobre estas descripciones: ISAF, DTD de XML de funciones y actividades, etc. Al parecer, la versión definitiva de la ISAF se presentará en el Congreso Internacional de Archivos de 2008.

- Fase 3: regulación de otras representaciones (lugares, temas, tipos documentales y tipos de materiales, etc.).

Teniendo en cuenta los períodos necesarios para la elaboración de estándares de contenido en otros casos, se considera que, para completar la fase inicial, se necesitarían unos cuatro o cinco años de desarrollo.

\section{c) Organización y funcionamiento}

Se propone que la estructura organizativa de desarrollo y mantenimiento de las NEDA incluya:

- Un Comité de Normas Españolas de Descripción Archivística: órgano colegiado integrado por no más de once expertos en descripción archivística, encargado de la redacción del proyecto y del desarrollo y mantenimiento de las NEDA.

- Una Secretaría: unidad administrativa permanente de apoyo al Comité, encargada de la gestión administrativa, la documentación y archivo, el mantenimiento del sitio Web de las NEDA, la conexión entre los miembros del Comité y los Grupos de Trabajo, etc.

- Varios Grupos de Trabajo: equipos de trabajo especializados, cada uno de ellos integrado por cuatro o cinco expertos, encargados por el Comité de elaborar propuestas técnicas concretas sobre la normalización de la descripción de determinados tipos de recursos archivísticos (gráficos, audiovisuales y electrónicos) u otras cuestiones específicas.

Dado que las NEDA serán normas técnicas para todos los archivos y archiveros, toda la Comunidad Archivística deberá estar implicada en su desarrollo y mantenimiento, por lo que deberá garantizarse:

- La participación formal de los Grupos de Trabajo que se establezcan en el Sistema Estatal de Archivos, en los Sistemas Archivísticos de las Comunidades Autónomas y en las Asociaciones de Archiveros, a través de la invitación formal a remitir comentarios y propuestas a los borradores de trabajo que se vayan elaborando y difundiendo. 
- La participación formal de expertos en sistemas de información archivística, incluyendo empresas de aplicaciones comerciales de archivos, mediante la invitación formal a enviar comentarios y propuestas de cambio a los borradores de trabajo que se vayan preparando y difundiendo.

\subsection{ISAAR (CPF). Norma Internacional sobre los Registros de Autoridad de Archivos relativos a Instituciones, Personas y Familias}

La información puede consignarse bajo una o varias de las siguientes formas:

- Un texto narrativo.

- Un texto estructurado conforme a los elementos abajo descritos.

- Enlaces con los recursos relacionados.

Esta Norma sirve de guía para elaborar registros de autoridad de archivos que proporcionan descripciones de entidades (instituciones, personas y familias) asociadas a la producción y a la gestión de archivos.

- Esta Norma renuncia a establecer criterios para redactar otros puntos de acceso (temáticos, geográficos, funcionales, materiales, etc.).

- Las personas y entidades pueden manifestar, en cuanto a su redacción, tantas diferencias nacionales como los nombres de lugar o los conceptos temáticos.

- Igualmente, esta Norma afronta detalladamente cuestiones de control, de metadatos y de enlaces.

Los registros de autoridad de archivos se pueden utilizar para:

- Describir una institución, persona o familia como unidades dentro de un sistema de descripción archivístico.

- Controlar la creación y utilización de los puntos de acceso en las descripciones archivísticas.

- Documentar las relaciones entre diferentes productores de documentos y entre estas entidades y los documentos creados por ellas, y otros recursos que les conciernen.

\section{a) Identificadores y títulos de los recursos relacionados}

\section{Objetivo:}

Identificar de forma única los recursos relacionados y establecer el vínculo entre el registro de autoridad y la descripción de los recursos relacionados cuando estos existan.

\section{Regla:}

Reseñar los identificadores únicos/códigos de referencias y títulos de los recursos relacionados. Cuando sea necesario, proporcionar también los identificadores de cualquier descripción del recurso relacionado. 


\section{b) Tipos de recursos relacionados}

\section{Objetivo:}

Identificar los tipos de recursos asociados al que se está haciendo referencia.

\section{Regla:}

Precisar los tipos de recursos relacionados, p.e. documentos de archivo (fondos, series etc.), descripción de archivo, instrumentos de descripción, monografía, artículo de prensa, página Web, fotografía, colección de museo, película documental, registro de historia oral, etc.

\section{c) Naturaleza de las relaciones}

\section{Objetivo:}

Identificar la naturaleza de las relaciones entre la institución, persona o familia y el/los recurso(s) relacionado(s).

\section{Regla:}

Describir la naturaleza de las relaciones entre la institución, persona o familia y el recurso relacionado, p.e. productor, autor, sujeto, depositario, propietario de los derechos de autor, administrador, propietario, etc.

\section{d) Fechas de los recursos relacionados y relaciones}

\section{Objetivo:}

Proporcionar cualquier fecha pertinente relativa a los recursos relacionados y las fechas de la relación entre la institución, persona o familia y los recursos relacionados e indicar su relevancia.

\section{Regla:}

Facilitar cualquier fecha o fechas de interés relativas al recurso asociado y su relación con la institución, persona o familia y explicar el significado de esas fechas.

\subsection{ISAD (G). Norma de descripción de descripciones archivísticas}

Esta Norma establece lo siguiente:

- Proporciona una guía general para la descripción de descripciones archivísticas.

- Se utiliza en conjunción con normas nacionales, o como base para el desarrollo de éstas.

- El objetivo de la descripción es identificar y explicar el contexto y contenido del material archivístico para promover su accesibilidad. Esto se logra creando representaciones fiables y adecuadas, y organizándolas de acuerdo a modelos predeterminados.

- Las reglas identifican 26 elementos que pueden combinarse para constituir la descripción archivística.

- La estructura y contenido de la información en cada elemento deben ser formuladas de acuerdo con reglas nacionales. 
- En cuanto a reglas generales, pretender ser aplicables a descripciones de archivos, con independencia de su naturaleza o la extensión de la unidad de descripción.

- Las reglas no definen formatos de salida.

- Las reglas se apoyan en principios aceptados: respeto al origen, proceder de lo general a lo específico, descripción jerárquica y multinivel.

Igualmente, pueden extrapolarse las siguientes conclusiones:

- La Norma se define como de uso general, y declara explícitamente que debe ser completada por reglas nacionales, o servir de desarrollo a éstas. En la práctica, esta adaptación no se ha llevado a cabo. Sin embargo, resulta ejemplar la adecuación llevada a cabo por el Archivo General de la Nación de Colombia, para introducir elementos que la Norma no consideraba.

- La Norma incide en la representación del contenido, el contexto y la estructura de la información aunque, posteriormente, al definir sus elementos, adopta una posición débil.

- La Norma renuncia explícitamente a definir formatos de salida.

\subsection{ISDIAH. Norma Internacional para describir instituciones que custodian fondos de archivo}

Esta Norma proporciona las reglas generales para la normalización de la descripción de instituciones que conservan fondos de archivo, y permite asimismo:

- Proporcionar directrices prácticas para identificar y contactar con las instituciones que detentan los fondos de archivo, y acceder a los mencionados fondos y a los servicios que la institución ofrece.

- Crear directorios y listas autorizadas de instituciones que custodian fondos archivísticos.

- Establecer enlaces con listas autorizadas de bibliotecas y museos y desarrollar directorios comunes de instituciones que custodian el patrimonio cultural a nivel regional, nacional o internacional.

- Producir, también a nivel regional, nacional e internacional, estadísticas sobre las instituciones que detentan documentos de archivo.

\subsection{ISDF. Norma para elaborar descripciones de funciones de instituciones vinculadas con la producción y conservación de documentos}

La Norma ISDF determina el tipo de información que puede incluirse en descripciones de funciones y sirve de guía sobre la forma de integrar estas descripciones en un sistema de información archivística. El contenido de los elementos de información incluidos en las descripciones estará determinado por las reglas y convenciones que utilice la institución encargada de elaborarlas.

Esta norma consta de elementos de información, cada uno de los cuales contiene: 
- El nombre del elemento de descripción.

- Una exposición del objetivo del elemento de descripción.

- Una exposición de la(s) regla(s) aplicable(s) a cada elemento.

- Cuando corresponda, ejemplos que ilustran la aplicación de la(s) regla(s).

De igual forma, los párrafos sólo están numerados para facilitar las citas. Esta numeración no se debe utilizar para designar los elementos de la descripción o para establecer el orden o estructura de las descripciones.

Los elementos de descripción están organizados en cuatro áreas de información:

- Área de Identificación. Se incluye la información que identifica unívocamente la función y que define un punto de acceso normalizado.

- Área de Contexto. Se recoge información sobre la naturaleza y contexto de la función.

- Área de Relaciones. Se consignan y describen las relaciones con otras funciones.

- Área de Control. Se identifica de forma unívoca la descripción de una función y se incluye información sobre cómo, cuándo y por qué institución se creó y actualizó la descripción.

Además, esta Norma proporciona en el capítulo directrices sobre la vinculación de las descripciones de funciones con los registros de autoridad que describen los productores de documentos de archivo y con las descripciones de documentos de archivo. Hay que señalar que una descripción de función puede vincularse con tantos registros de autoridad o descripciones de documentos de archivo como sean necesarios. El análisis de las funciones de las instituciones es importante como base para muchas actividades de la gestión de documentos. Generalmente, se reconoce que las funciones son más estables que las estructuras administrativas, ya que frecuentemente se fusionan o se transfieren cuando se produce una reestructuración.

Por tanto, las funciones sirven apropiadamente:

- De base para la clasificación y descripción de los documentos.

- De base para la valoración documental.

- De herramienta para la recuperación y análisis de los documentos. 
Cuadro 1. Comparativa de normas de descripción archivística

\begin{tabular}{|c|c|c|c|c|}
\hline CARACTERÍSTICA & $\begin{array}{l}\text { ISAAR } \\
\text { (CPF) }\end{array}$ & $\begin{array}{l}\text { ISAD } \\
(\mathrm{G})\end{array}$ & ISDIAH & ISDF \\
\hline No establece criterios para redactar otros puntos de acceso & $\mathrm{x}$ & & & \\
\hline $\begin{array}{l}\text { Se identifican } 26 \text { elementos que pueden combinarse para constituir la descripción } \\
\text { archivística }\end{array}$ & & $X$ & & \\
\hline $\begin{array}{l}\text { Identifica y explica el contexto y el contenido del material archivístico para promover } \\
\text { su accesibilidad }\end{array}$ & & $\mathrm{X}$ & & \\
\hline No se definen formatos de salida & & $\mathrm{X}$ & & \\
\hline Elabora estadísticas acerca de las instituciones que custodian documentos de archivo & & & $\mathrm{X}$ & \\
\hline $\begin{array}{l}\text { Crea directorios y listas autorizadas de instituciones que custodian fondos } \\
\text { archivísticos }\end{array}$ & & & $\mathrm{X}$ & \\
\hline $\begin{array}{l}\text { El contenido de los elementos de información incluidos en las descripciones estará } \\
\text { determinado por las reglas de la institución }\end{array}$ & & & & $x$ \\
\hline Los elementos de descripción están organizados en cuatro áreas de información & & & & $\mathrm{x}$ \\
\hline Los párrafos solo están numerados para facilitar las citas & & & & $\mathrm{x}$ \\
\hline La Norma consta de cuatro elementos de información & & & & $\mathrm{x}$ \\
\hline Se afrontan detalladamente cuestiones de control, metadatos y enlaces & $\mathrm{x}$ & & & \\
\hline $\begin{array}{l}\text { Promulga los Registros de Autoridad de Archivos relativos a Instituciones, Personas y } \\
\text { Familias }\end{array}$ & $x$ & & & \\
\hline Proporciona una guía general para la descripción de descripciones archivísticas & & $\mathrm{x}$ & & \\
\hline Describe instituciones que custodian fondos de archive & & & $\mathrm{x}$ & \\
\hline $\begin{array}{l}\text { Elabora descripciones de funciones de instituciones vinculadas con la producción y } \\
\text { conservación de documentos }\end{array}$ & & & & $\mathrm{X}$ \\
\hline
\end{tabular}

Fuente: elaboración propia

\section{Principales Normas de descripción bibliotecaria}

Desde los inicios del siglo XX, distintas organizaciones y grupos de estudiosos comenzaron a comprender la importancia de establecer normas que orientaran el desarrollo de los procesos, productos y servicios. Un momento importante para el desarrollo de la normalización fue cuando, en 1926, algunos organismos nacionales de normalización fundaron la Federación Internacional de Asociaciones Nacionales de Normalización (ISA), con la finalidad de promover el comercio internacional mediante la estandarización de los procesos de producción y los productos. La ISA puede considerarse el precedente inmediato de la actual Organización Internacional de Normalización (ISO, en inglés), creada después de la Segunda Guerra Mundial (Pérez, 2005).

La actividad de normalización se ha convertido actualmente en una necesidad del desarrollo de las organizaciones. Las bibliotecas y centros de documentación no escapan a esta realidad, ya que no se concibe hablar de redes, intercambio cooperativo, bibliotecas virtuales y acceso a recursos compartidos, sin que existan normas o estándares que sistematicen y regulen la su ejecución. Ahora bien, ¿existen diferencias entre normas y estándares? ¿Presentan estos términos significados diferentes o se trata de un mismo concepto con distintas dimensiones? En este punto es 
oportuno hacer un alto para definir a qué se hará referencia cuando se emplee uno u otro término y establecer cómo se presenta esta problemática en la literatura consultada (Fernández Valdés y Sánchez, 2005).

Por normalización se entiende toda actividad colectiva dirigida a establecer e implementar normas para definir los requisitos que deben cumplir bienes, servicios y procedimientos. El fin primordial de la normalización es solucionar situaciones repetitivas y unificar criterios, al posibilitar la utilización de un lenguaje común en un campo de actividades concretas. La normalización consiste en la elaboración, difusión y aplicación de normas de con carácter voluntario. La normalización es un proceso dinámico y continuo imprescindible para el mejor funcionamiento de toda organización y la obtención de buenos resultados económicos (Valdés Abreu, 1996).

Un glosario de término de normalización consultado en Internet establece: Estandarizar (anglicismo): Normalizar. Convertir algo en Norma. Regular por medio de una Norma, de un estándar. Fijar como la Norma aceptada, que además debe obedecerse. Según Louis-Jean Rosseau, Secretario General de la Red Internacional de Neología y Terminología en Francia, en planificación lingüística, los conceptos de normalización y estandarización se oponen. Se habla de normalización cuando se trata de opciones terminológicas oficiales realizadas por un organismo que goza de autoridad reconocida (la ISO, por ejemplo). El concepto de normalización se refiere a una orientación del uso más o menos fuerte, según sean la naturaleza y la influencia del organismo que expide. En cambio, el concepto de estandarización se aplica a la autorregulación de la terminología por el consenso implícito de los hablantes en un medio profesional o a la "normalización de facto" (Termilat, 2005).

La Norma es un documento establecido por consenso y aprobado por un organismo reconocido, que ofrece para uso común y repetido, reglas y lineamientos para la realización de las actividades o la obtención de sus resultados, con la finalidad de lograr el mejor orden en un contexto determinado. Deben basarse en resultados consolidados por la ciencia, la tecnología y la experiencia, y están destinadas a la promoción de beneficios para la comunidad.

Los estándares son normas que orientan sobre los requisitos indispensables que debe cumplir determinado proceso, producto o servicio para alcanzar sus objetivos de calidad. El Glosario de ALA sobre Bibliotecología y Ciencias de la Información define "estándares" como "criterios, pautas, normas... Criterios por los que pueden medirse o evaluarse los servicios y programas bibliotecarios. Establecidos por organizaciones profesionales, corporaciones acreditadas $u$ organismos estatales, los criterios pueden reflejar de diversos modos, un mínimo o ideal, un procedimiento modélico, una medida cuantitativa o una evaluación cualitativa" (García Martín, 1999).

El análisis de las definiciones y criterios expuestos indica que existe una falta de consenso para definir el concepto de estandarización, los criterios se dividen. Mientras algunos piensan que se trata de un problema de traducción del término de una lengua a otra con un mismo significado, otros asumen que la estandarización es un proceso particular, que puede realizarse por instituciones expertas en determinados temas, pero que, necesariamente, los estándares no tienen que aprobarse por un organismo internacional o nacional dedicado a la actividad de normalización (Fernández Valdés y Sánchez, 2005).

\subsection{ISBD}

La Descripción Bibliográfica Internacional Normalizada (ISBD) pretende servir como Norma principal para la promoción del control bibliográfico universal, en definitiva, para que la información bibliográfica básica de todos los recursos publicados en todos los países se encuentre disponible universal y rápidamente, en una forma aceptable internacionalmente. Desde el principio, el principal objetivo de la ISBD ha sido ofrecer coherencia con vistas al intercambio de información bibliográfica.

La ISBD es la Norma que determina los elementos de datos que se deben registrar o transcribir en un orden específico como base para la descripción del recurso que se está catalogando. Además, emplea puntuación prescrita como medio para reconocer y mostrar estos elementos de datos y hacerlos comprensibles con independencia de la lengua de descripción. En 2009 se ha publicado por IFLA la nueva Declaración de Principios Internacionales de Catalogación. La quinta sección de estos principios, que sustituyen y amplían los Principios de París de 1961, está dedicada a la descripción bibliográfica, donde se establece que "Los datos descriptivos deben basarse en una Norma acordada internacionalmente. Una nota a pie de página identifica la ISBD como el estándar 
para la comunidad bibliotecaria, ya que la declaración de principios no está destinada solo para bibliotecas, sino también para archivos, museos y otras comunidades.

La Descripción Bibliográfica Internacional Normalizada (ISBD) especifica los requisitos para la descripción e identificación de los recursos publicados que pueden constituir las colecciones de las bibliotecas. También se ocupa de los recursos para su uso por personas con discapacidad visual (Ej. En forma legible visual o en forma de relieve), así como los publicados para distribución limitada o para venta bajo demanda. La ISBD determina los elementos de datos que se registrarán o transcribirán en un orden específico, como base de la descripción del recurso que se está catalogando, y emplea una puntuación prescrita como medio de reconocimiento y visualización de los elementos de datos, haciéndolos comprensibles independientemente de la lengua de descripción.

Las disposiciones de la ISBD se destinan, en primer lugar, a los registros bibliográficos producidos por las agencias bibliográficas nacionales y, en segundo lugar, a los de otros centros catalogadores.

\section{a) Objetivos}

- Proporcionar especificaciones para una catalogación descriptiva compatible a nivel mundial, con el fin de facilitar el intercambio internacional de registros bibliográficos entre agencias bibliográficas nacionales y dentro de la comunidad internacional bibliotecaria y de la información.

- Hacer que los registros de diferentes fuentes sean intercambiables, de manera que los registros elaborados en un país se puedan asimilar fácilmente en los catálogos de bibliotecas o en listas bibliográficas de otros países.

- Ayudar a la interpretación de los registros por encima de las barreras lingüísticas, de modo que registros producidos para usuarios de una lengua puedan ser interpretados por usuarios de otra.

- Facilitar la conversión de los registros bibliográficos a formato electrónico.

- Mejorar la portabilidad de los datos bibliográficos al entorno de la Web Semántica y la interoperabilidad con otras normas de contenido.

La ISBD se utiliza para crear una descripción de un conjunto de recursos que tienen las mismas características tanto con respecto al contenido intelectual como al formato físico, es decir, una edición. Se puede identificar una edición por una mención de edición en el recurso o por la información proporcionada por el editor. Una diferencia importante en cualquiera de los elementos entre dos recursos indica que están involucradas diferentes ediciones y, por tanto, se requieren descripciones separadas. Un cambio en la identidad del distribuidor no constituye una diferencia importante. Una descripción ISBD representa una copia completa de un recurso publicado.

Los recursos pueden ser publicados en una única parte o recursos multiparte, finitos o destinados a ser finitos, o con la intención de ser continuados en el tiempo y pueden ser emitidos en un momento dado o en periodos sucesivos. Un recurso de una única parte es un recurso que se publica como una única unidad física. Los recursos multiparte se catalogan utilizando una descripción en varios niveles o una descripción de todo el recurso en un solo nivel. En algunos casos las partes de un recurso multiparte se pueden describir también como recursos en una única parte separada.

Las ISBD se componen de diversas áreas de descripción:

- Área de forma del contenido y de tipo de medio.

- Área de título y mención de responsabilidad.

- Área de edición. 
- Área específica de material o tipo de recurso.

- Área de publicación, producción, distribución.

- Área de descripción física.

- Área de serie y recurso monográfico multiparte.

- Área de notas.

- Área de identificador del recurso y condiciones de disponibilidad.

\section{b) Historia de las ISBD}

Algunos hitos importantes del desarrollo de las ISBD son los siguientes:

- La Descripción Bibliográfica Internacional Normalizada se remonta a 1969, cuando el Comité sobre Catalogación de IFLA auspició una Reunión Internacional de Expertos en Catalogación. Esta reunión elaboró una resolución en la que proponía la creación de Normas que regularizasen la forma y el contenido de las descripciones bibliográficas.

- La primera de las ISBD fue la Descripción Bibliográfica Internacional Normalizada para Publicaciones Monográficas (ISBD (M)), que apareció en 1971.

- La Descripción Bibliográfica Internacional Normalizada para Publicaciones Seriadas (ISBD(S)) se publicó en 1974.

- En 1975, el Comité Conjunto Directivo para la Revisión de las Reglas de Catalogación Angloamericanas propuso al Comité sobre Catalogación de IFLA acometer el desarrollo de una Norma de descripción bibliográfica internacional normalizada general adecuada para todos los tipos de materiales bibliotecarios. Como resultado, en 1977 se publicó la $\operatorname{ISBD}(G)$. La $\operatorname{ISBD}(\mathrm{M})$ fue entonces revisada a fin de adaptarla a la $\operatorname{ISBD}(G)$ y, en 1978, se publicó la "Primera edición estándar normalizada revisada".

- En 1977 se publicaron la ISBD(CM) para materiales cartográficos, la ISBD(NBM) para materiales no librarios y una revisión de la $\operatorname{ISBD}(S)$ para publicaciones seriadas.

- En 1980 se publicaron la $\operatorname{ISBD}(\mathrm{A})$ para publicaciones monográficas antiguas (Anticuaria) y la ISBD(PM) para música impresa.

- Las ISBD se reeditaron de la siguiente manera: la ISBD(M), la ISBD(CM) y la ISBD(NBM) se reeditaron en 1987; la ISBD(S), en 1988; la ISBD(CF) para archivos de ordenador se publicó en 1990; la ISBD(A) y la ISBD(PM), en 1991; y la ISBD(G), en 1992. Posteriormente, la ISBD(CF) se convirtió en ISBD(ER) para recursos electrónicos, publicándose en 1997.

- En 1992, la Sección sobre Catalogación de la IFLA, en cooperación con la Sección sobre Clasificación e Indización, crearon un Grupo de Estudio sobre los Requisitos Funcionales para Registros Bibliográficos (FRBR). Una consecuencia inmediata de este desarrollo fue la decisión de suspender la mayoría de los trabajos de revisión de las ISBD, mientras que el Grupo de Estudio FRBR llevaba a cabo la tarea de "recomendar un nivel básico de funcionalidad y unos 
requisitos básicos de información para los registros creados por las agencias bibliográficas nacionales".

- En este proyecto se ha llevado a cabo la revisión de la ISBD(S), que se convirtió en $\operatorname{ISBD}(\mathrm{CR})$ para publicaciones seriadas y otros recursos continuados y que fue publicada en 2002, tras las reuniones celebradas para armonizar la ISBD(S) con las directrices del ISSN y con las Reglas de Catalogación Angloamericanas, 2. ${ }^{a}$ edición. La ISBD(M) revisada se publicó también en 2002 y la revisión de la $\operatorname{ISBD}(\mathrm{G})$ lo hizo en 2004. La ISBD(CM) y la ISBD(ER) siguieron el proceso de revisión mundial y fueron modificadas de acuerdo con él, pero no se completó el proceso de revisión en ese momento, ya que se comenzó a trabajar en una ISBD consolidada.

- Tras varias revisiones y un periodo de revisión mundial, una nueva Área 0, denominada Área de Forma del Contenido y de Tipo de Medio con sus elementos del mismo nombre, fue aprobada en 2009.

\subsection{Los Formatos MARC}

El formato MARC (Machine Readable Cataloging) o Catalogación Legible por Máquina permite el intercambio de información, el acceso a catálogos colectivos y la catalogación compartida entre bibliotecas.

Presenta tres tipologías:

- MARC21.

- UNIMARC.

- IBERMARC.

El formato MARC es una herramienta importante que tiene como objetivo conseguir un grado óptimo de normalización, compatibilidad y transferencia de la información bibliográfica, legible por ordenador. Permite, además, la cooperación y compartir recursos y servicios entre bibliotecas.

- 001 - Número de control.

- 003 - Identificador del número de control.

- 005 - Fecha y hora de la última transacción.

- 006 - Códigos de información de longitud fija. Características del material adicional.

- 007 - Campo fijo de descripción física.

- 008 - Códigos de información de longitud fija.

\section{a) MARC21}

La traducción se ha hecho basándose en el texto inglés MARC 21 Format for Bibliographic Data, incluida su Actualización n.ำ 12, de octubre de 2010, y en la edición de IBERMARC bibliográfico 6. a ed. 2001. De aquí en adelante, se van incorporando las nuevas actualizaciones del Formato.

Se ha creado un vocabulario estable de palabras inglés-español, para el que se ha seguido la terminología de las traducciones de ISBD, PIC, FRAD y MuldiCat, a la que se han añadido nuevas palabras 0 
acepciones de palabras, que se pondrán en un documento en la Web para consulta de las personas interesadas.

\section{b) UNIMARC}

En 1977, el Grupo de Trabajo sobre designadores de contenido de la IFLA publicó el Formato UNIMARC, que permite el intercambio de registros bibliográficos entre diferentes órganos bibliográficos nacionales, mediante un lenguaje común. De esta forma, concluyeron la tarea de elaboración de una Norma de ámbito internacional referente a los identificadores del contenido de registros legibles en soporte informático que resolvía las incompatibilidades entre varios formatos MARC nacionales.

\section{c) IBERMARC}

El formato IBERMARC sigue el formato MARC en sus líneas generales y en su filosofía y se aparta de él en algunos puntos concretos relativos, sobre todo, a la práctica catalográfica tradicional española. Un registro IBERMARC se compone de tres elementos: la estructura del registro, los identificadores de contenido y el contenido del registro. Además, una de las funciones de la Biblioteca Nacional es la elaboración de la normativa relacionada con la descripción de registros bibliográficos, de autoridades y de fondos y localizaciones. Dentro de esta normativa se encuentra la relacionada con el tratamiento de registros que permita y facilite el intercambio de información bibliográfica en soporte legible por ordenador. La norma internacional ISO 2709, basada a su vez en la ANSI Z39.2 para la estructuración lógica de la información bibliográfica, es el sustento de los Formatos MARC de intercambio de información bibliográfica, de autoridades y de fondos y localizaciones.

La Biblioteca Nacional ha realizado un gran esfuerzo para preparar las distintas versiones de los formatos IBERMARC para registros de autoridad, para registros bibliográficos y para registros de fondos y localizaciones, guardando la coherencia entre aquellos elementos comunes a dos o más formatos. A partir del año 1992, la Biblioteca Nacional empezó a difundir, como borrador o en edición resumida, los distintos formatos. En 1999 se editó el Formato IBERMARC para registros de autoridad. Posteriormente, se incorporó el Formato MARC 21 y el Formato IBERMARC para registros de fondos y localizaciones.

\section{d) Las Reglas de Catalogación Españolas}

La Biblioteca Nacional de España (BNE) pone a disposición de bibliotecarios y usuarios, una de las herramientas fundamentales para la normalización catalográfica y la adecuada gestión de los procesos técnicos bibliográficos. De esta forma, siguiendo la línea de actuación que la Biblioteca Nacional tiene de poner accesibles en Internet todas las herramientas de trabajo, al igual que se ha hecho con los formatos de codificación, ha realizado la digitalización de todos los códigos que han normalizado, hasta la actualidad, el proceso de catalogación en España con el objeto de ponerlos a disposición del público en general y, en especial, para el estudio e investigación de los profesionales de la Biblioteconomía; y para que todas las bibliotecas tengan a su disposición en línea el código vigente de aplicación, pretendiendo con ello fomentar una mayor normalización y cooperación.

Se divide en las siguientes áreas de descripción bibliográfica:

- Área de título y mención de responsabilidad.

- Área de edición.

- Área de datos específicos de la clase de documento.

- Área de publicación, distribución, etc.

- Área de descripción física. 
- Área de serie.

- Área de notas.

- Área del número normalizado y de las condiciones de adquisición.

- Documentos en varias unidades físicas.

\section{Conclusiones}

Tras la investigación realizada, pueden extrapolarse las siguientes premisas:

- Las normas de descripción archivística y bibliotecaria han evolucionado de forma sustancial durante los últimos años. Esta progresiva evolución ha estado sustentada por el desarrollo de las Tecnologías de la Información y de la Comunicación (TIC). Cada Unidad de Información (archivo, biblioteca, centro de documentación) ha procurado tener visibilidad en Internet con el objetivo de interactuar con el usuario y facilitarle una primera aproximación "en línea" a sus fondos documentales. Esta primera información servirá al usuario para conocer dónde se encuentra el documento preceptivo para satisfacer sus necesidades de información y responder a sus intereses investigadores.

- El profesional de la documentación tiene asignadas actividades básicas como localizar la información, analizarla y tratarla para aumentar su valor para quien la tiene que utilizar, y, al mismo tiempo, gestionarla y convertirla en accesible para los potenciales usuarios. Estos profesionales tienen que adquirir competencias relacionadas con la planificación y la gestión, así como las inherentes a las áreas de actividad en las que desarrollan su trabajo.

- Por lo que respecta a la formación académica de los profesionales de la documentación, en la mayoría de las convocatorias públicas de oposiciones no se solicita la titulación pertinente de Grado en Información y Documentación, Licenciado en Documentación o Diplomado en Biblioteconomía y Documentación. Esto supone una situación de agravio comparativo, ya que pueden presentarse Licenciados e Ingenieros de cualquier área de conocimiento. En este sentido, sería muy conveniente la creación del Colegio Oficial de Archiveros, Bibliotecarios y Documentalistas, lo que supondría un mayor reconocimiento social y profesional de estos titulados.

- Igualmente, tanto archiveros como bibliotecarios tienen que conocer qué tipología de servicios utilizan los usuarios; con qué frecuencia lo hacen; las razones que les llevan a usarlos, las estrategias de búsqueda que emplean; así como sus conocimientos de Tecnologías de la Información y de la Comunicación (TIC).

- Las Normas de descripción archivística se utilizan y adaptan según las necesidades de cada archivo. En este sentido, hay que tener muy en cuenta las especiales características que presenta el documento de archivo, mucho más complejo en su descripción que las tipologías documentales existentes en una biblioteca. Aunque suelen respetarse las Normas de descripción tanto en archivos como en bibliotecas, también se da el caso de que algunos centros adaptan dichas Normas al fondo documental que poseen.

- Los profesionales que trabajan en archivos y bibliotecas también necesitan unas Normas de descripción cohesionadas y coherentes para ordenar las diferentes tipologías documentales existentes con el fin de satisfacer las necesidades informacionales de los usuarios. Si los usuarios están satisfechos, esto redundará en la calidad del servicio prestado en el archivo, la biblioteca o el centro de documentación de que se trate. 
- Sin embargo, en multitud de ocasiones, estas Normas de descripción requieren de cambios continuos que, a priori, producen un aumento del volumen de trabajo de los profesionales. En la mayoría de las ocasiones, también conllevan un coste económico que no todas las Unidades de Información están dispuestas a asumir.

- También, la mayoría de las veces, hay que coordinarse con otras instituciones de ámbito internacional para la redacción de unas Normas de descripción comunes y coherentes que promuevan la coordinación de equipos de trabajo y satisfagan las necesidades de información de los usuarios.

- Además, es muy importante la gestión del tiempo, tanto de los usuarios como de los profesionales que trabajan en las distintas Unidades de Información.

- Existen dificultades para establecer una normalización a nivel nacional que contemple unos campos de descripción comunes y el complicado proceso de identificarlos dadas las características y conceptos que intervienen en la descripción de las agrupaciones documentales y sus contenidos.

- Dados los continuos cambios en las Normas de descripción archivística y bibliotecaria, los profesionales se encuentran en una situación de "actualización constante" que, en muchas ocasiones, tienen que transmitirlas a los usuarios para que éstos satisfagan sus necesidades de información.

- Por regla general, los usuarios están más habituados a consultar las tipologías documentales existentes en una biblioteca que las de un archivo o centro de documentación. En muchas ocasiones, la consulta de los documentos de archivo se produce por usuarios especializados (docentes, investigadores, profesionales).

\section{Bibliografia}

BARBADILLO ALONSO, J. (2011). Las Normas de descripción archivística. Qué son y cómo se aplican. Gijón: Trea.

BEARMAN, D. (1989). "Description standards: a framework for action". The American archivist. 52: 4, pp. 5-17.

BOLT, N. (1989). Standards for public libraries. The library trustee. 4th ed. Chicago: American Library Association, p. 105.

BONAL ZAZO, J. L. (2000). "La normalización: base del análisis documental en los archivos", Scire, 6 (1), pp. 55-75.

COX, R. J. (1994). Standardizing archival practices: a tool for the information age. International Congress of Archives (12.ำ 1992. Montreal). Proceedings. Archivum. 39.

CRAWFORD, W. (1986). Technical standards: an introduction for librarians. New York: Knowledge Industry Publications.

DELGADO GÓMEZ, A. (2003). Normalización de la descripción archivística: introducción a Encoded Archival Description (EAD). Cartagena: Archivo Municipal.

FERNÁNDEZ VALDÉS, M. ㄹ DE LAS M.; ALFONSO SÁNCHEZ, I. R. (2005). "Estado actual de la normalización y la estandarización en las Bibliotecas sobre Ciencias de la Salud", Acimed, 13 (5). Disponible en:

http://bvs.sld.cu/revistas/aci/vol13 5 05/aci03505.htm [Consultado: 24 de junio de 2013].

GARCíA GUTIÉRREZ, A. (1985). "Normalización general y documental: concepto, historia e instituciones". Bol Doc Cienc Inf, 9, pp. 55-96.

GARCÍA MARTÍN, M. A. (1999). Estándares para Bibliotecas en Ciencias de la Salud. Disponible en: http://sedic.es/estandar.pdf [Consultado: 26 de junio de 2013].

GONZÁLEZ, P. (1992). La normalización en la descripción de archivos: un movimiento internacional. Jornadas sobre metodología para la identificación y valoración de fondos documentales de las Administraciones Públicas (1. ${ }^{\text {a }} 1991$. Madrid). Actas. Madrid: Dirección de Archivos Estatales. 
GUERCIO, M. - (1994). Models for fostering the development of standard practices within a region or a particular country. International Congress on Archives (12. 1992. Montreal). Proceedings. Archivum. 39.

HAWORTH, K. M. (1994). Standardizing archival description. International Congress on Archives (12.. 1992. Montreal). Proceedings. Archivum. 39.

MUÑOZ FELIU, M. C. (1995). "ISAD(G): hacia un estándar internacional de descripción archivística". Métodos de información, $2: 8$.

PÉREZ, C. (2002). Normalización terminológica: esfuerzos de estandarización e instituciones de normalización. Disponible en: http://elies.rediris.es/elies18/321.html [Consultado: 22 de junio de 2013].

SZARY, R. V. (1990). "Archival applicability of external guidelines for data structures". The American archivist, 53: 1, pp. 52-54.

TARRAUBELLA I MIRABET, X. (1998). "Els arxius i els seus usuaris". Lligall, 12, pp. 190-204.

TERMILAT (1999). Normalización, estandarización. Disponible en: http://www.termilat.info/env347.htm [Consultado: 27 de junio de 2013].

VALDÉS ABREU, M. C. (1996). "Las Normas ISO relacionadas con la documentación y la información", Acimed, 4 (3). Disponible

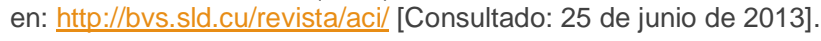

WEBER, L. B. (1989). "Archival description standards: concepts, principles and methodologies", American Archivist, 52 (4).

WORKING GROUP ON STANDARDS FOR ARCHIVAL DESCRIPTION (1989). "Report of the Working Group on Standards for Archival Description". American archivist, 52 (4), pp. 456-461.

\section{Datos del autor}

\section{Carlos Oliva Marañón}

Profesor de la Universidad Rey Juan Carlos. Departamento de Ciencias de la Ciencias de la Comunicación y Lengua Española. Doctor en Ciencias de la Comunicación. Sus líneas de investigación se centran en las Fuentes de Información en Comunicación, así como en el análisis de los Grados en Humanidades y Ciencias Sociales de las Universidades de España conforme al Espacio Europeo de Educación Superior (EEES).

carlos.oliva.maranon@uric.es

Recibido - Received: 2013-07-23

Aceptado - Accepted: 2013-12-30

\section{$($ (c) $)$ EY}

This work is licensed under a Creative Commons Attribution 4.0

United States License.

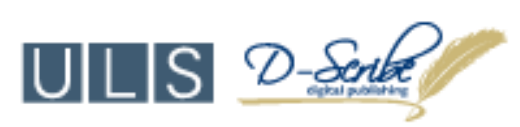

This journal is published by the University Library System of the University of Pittsburgh as part of its D-Scribe Digital Publishing Program and is cosponsored by the University of Pittsburgh Press. 